\title{
Lumped Element Modeling of CMUT Arrays in Collapsed Mode
}

\author{
Elif Aydogdu* ${ }^{* \dagger}$, Alper Ozgurluk ${ }^{\S}$, Abdullah Atalar ${ }^{\ddagger}$, and Hayrettin Koymen ${ }^{\ddagger}$ \\ * ASELSAN, TURKEY \\ ${ }^{\ddagger}$ Bilkent University, Electrical and Electronics Engineering Department, TURKEY \\ $\S$ University of California at Berkeley, Electrical Engineering and Computer Sciences Department, CA, USA \\ †e-mail: aelif@ee.bilkent.edu.tr
}

\begin{abstract}
This study focuses on modeling collapsed mode operation of CMUT arrays, and obtaining a small signal lumped element model for collapsed mode operation. Having the large signal model for single CMUT from previous studies, the mutual radiation impedance is presented for the collapsed mode, and a large signal model for a CMUT array is obtained for simulating the operation in both uncollapsed and collapsed modes. For faster computation, a small signal model for a CMUT cell is derived by linearizing the collapsed mode operation at a given bias point, and the computation time is reduced significantly. Using this model we are able to simulate a large array of collapsed CMUT cells.
\end{abstract}

\section{INTRODUCTION}

Lumped element modeling provides a feasible method to simulate a large CMUT array of finite length. Using this method, the performance of a group of CMUT cells operating in the conventional uncollapsed mode was predicted with a low computational cost [1]. This study aims at modeling of a collapsed mode CMUT array in a similar manner. In our previous studies we have obtained a parametric, large signal lumped element model for the single CMUT in collapsed mode [2].

In the model, through and across variables are defined as rms quantities. The instantaneous electrical capacitance of a single cell, $C$, is defined as a function of the plate displacement, $x_{R}{ }^{1}$. On the mechanical side, the $r m s$ velocity, $v_{R}$, is the through variable, and the force, $f_{R}$, is the across variable. The plate compliance is modeled with a capacitance of $C_{R m}$, and the plate mass is modeled with an inductance of $L_{R m}$, all defined in rms quantities.

For the uncollapsed mode, simple approximations allowed us to express $C$ and $f_{R}$ as analytical functions of $x_{R}$ [3], and the compliance has a fixed value. However, in the collapsed mode, analytical expressions cannot be obtained for capacitance and electrical force. The compliance is no longer a constant as it changes instantaneously with the plate deflection. The compliance, the electrical force, the electrical capacitance and the radiation impedance of the collapsed mode model are all expressed as functions of instantaneous plate

\footnotetext{
${ }^{1}$ The root-mean-square (rms) displacement is defined as a lumped variable as $x_{R}=1 /\left(\pi a^{2}\right) \sqrt{\int_{0}^{a} 2 \pi r x^{2}(r) d r}$ where $x(r)$ is the radius dependent displacement and $a$ is the plate radius.
}

displacement. This increases the computation cost of collapsed mode simulation.

In this work, the model is simplified in order to obtain a small signal model with lower computation cost. In order model arrays, the mutual radiation impedance is also calculated for the collapsed mode operation, and it is introduced to the model to express the forces between every CMUT cell pair in the array.

\section{Small Signal Model}

The small signal operation of a CMUT can be modeled simply using only linear components, by linearizing the equivalent circuit around an operation point determined by the applied bias voltage $V_{D C}$ and the static medium pressure.

The small signal model for the collapsed mode operation is the same as the uncollapsed mode, with the exception of an additional capacitance, $C_{R m_{-} a c}$, which stands for the deflection dependence of the compliance.

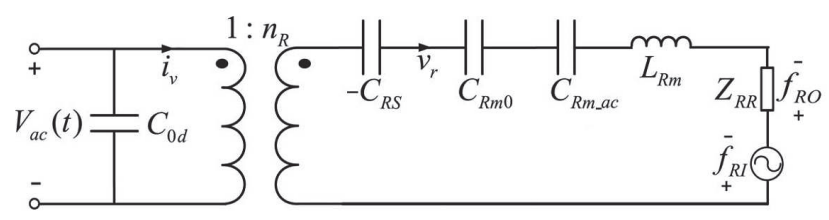

Fig. 1. The small signal circuit model for collapsed mode CMUT

On the electrical side, the small signal capacitance is assumed to be fixed at the capacitance of the DC deflection:

$$
C_{0 d}=C\left(X_{R}\right)
$$

The voltage-electrical force relation is handled through the electromechanical transformer and the spring softening capacitance, $C_{R S}$. They are calculated by linearizing the electrical force, $f_{R}$ around $X_{R}$, the rms deflection at the applied bias voltage. We write

$$
f_{R}=\frac{d E_{e l}}{d x_{R}}=\frac{V^{2}}{2} \frac{d C\left(x_{R}\right)}{d x_{R}}
$$

where

$$
V(t)=V_{D C}+v_{a c}(t)
$$


Keeping the first two terms of Taylor expansion:

$$
f_{R}=\frac{V^{2}}{2}\left(\left.\frac{d C\left(x_{R}\right)}{d x_{R}}\right|_{x_{R}=X_{R}}+\left.x_{r} \frac{d^{2} C\left(x_{R}\right)}{d x_{R}^{2}}\right|_{x_{R}=X_{R}}\right)
$$

where $x_{r}=x_{R}-X_{R}$ is the small-signal ac displacement. For $V^{2} \simeq V_{D C}^{2}+2 V_{D C} v_{a c}(t)$, we find:

$$
\begin{aligned}
f_{R} \simeq & \left.\frac{V_{D C}^{2}}{2} \frac{d C\left(x_{R}\right)}{d x_{R}}\right|_{x_{R}=X_{R}}+\left.V_{D C} v_{a c} \frac{d C\left(x_{R}\right)}{d x_{R}}\right|_{x_{R}=X_{R}} \\
& +\left.\frac{V_{D C}^{2} x_{r}}{2} \frac{d^{2} C\left(x_{R}\right)}{d x_{R}^{2}}\right|_{x_{R}=X_{R}}
\end{aligned}
$$

The first term is the DC force which will not appear in the small signal model. Therefore, the ac force, $f_{r}$, arising from ac excitation is given by

$$
f_{r}=n_{R} v_{a c}+\frac{x_{r}}{C_{R S}}
$$

where

$$
\begin{aligned}
n_{R} & =V_{D C} C^{\prime}\left(X_{R}\right) \\
C_{R S} & =\frac{2}{V_{D C}^{2} C^{\prime \prime}\left(X_{R}\right)}
\end{aligned}
$$

In the collapsed mode, the compliance changes instantaneously with the changing displacement, and the restoring force of the plate is also nonlinearly dependent on the displacement. Therefore, the restoring force, $f_{S}$, should be linearized around $X_{R}$ :

$$
\begin{aligned}
f_{S} & =\frac{x_{R}}{C_{R m}\left(x_{R}\right)} \\
& \left.\simeq \frac{x_{R}}{C_{R m}\left(x_{R}\right)}\right|_{x_{R}=X_{R}}+\left.x_{r} \frac{d\left(x_{R} / C_{R m}\left(x_{R}\right)\right)}{d x_{R}}\right|_{x_{R}=X_{R}} \\
& =\frac{X_{R}}{C_{R m 0}}+x_{r}\left(\frac{1}{C_{R m 0}}-\frac{X_{R}}{C_{R m 0}^{2}} C_{R m}^{\prime}\left(X_{R}\right)\right)
\end{aligned}
$$

Hence the small signal restoring force, $f_{s}$, is

$$
f_{s}=x_{r}\left(\frac{1}{C_{R m 0}}+\frac{1}{C_{R m_{-} a c}}\right)
$$

with

$$
\begin{aligned}
C_{R m 0} & =C_{R m}\left(X_{R}\right) \\
C_{R m_{-} a c} & =-\frac{C_{R m 0}^{2}}{X_{R} C_{R m}^{\prime}\left(X_{R}\right)}
\end{aligned}
$$

The inductance in the rms equivalent circuit is equal to the mass of the plate:

$$
L_{R m}=\pi a^{2} t_{m} \rho
$$

where $a, t_{m}, \rho$ are the CMUT plate radius, thickness and density, respectively.

Although the radiation impedance is instantaneously dependent on the contact radius, in the small signal model, we use the value at the contact radius generated by the DC bias.
A. Comparison of Simulations with Large and Small Signal Models

To check our small-signal model, both large signal and small signal simulations are carried out in water at $1 \mathrm{~atm}$ for a single CMUT cell with the parameters given in Table I. The resulting

\begin{tabular}{|l|l|l|l|}
\hline$E$ & $v$ & $\epsilon_{r}$ & $\rho$ \\
\hline $110 \mathrm{GPa}$ & 0.27 & 5.4 & $3.1 \mathrm{~g} / \mathrm{cm}^{3}$ \\
\hline \hline$a$ & $t_{m}$ & $t_{i}$ & $t_{g}$ \\
\hline $30 \mu \mathrm{m}$ & $1.2 \mu \mathrm{m}$ & $0.4 \mu \mathrm{m}$ & $0.2 \mu \mathrm{m}$ \\
\hline
\end{tabular}

TABLE I

PARAMETERS OF THE CMUT CELL USED IN SIMULATIONS

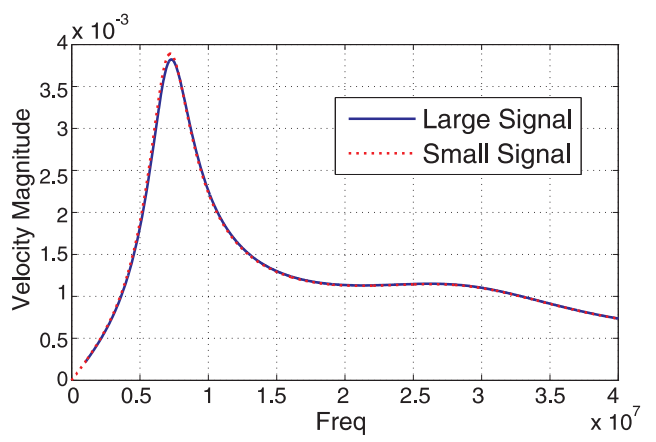

Fig. 2. Simulations with small and large signal models for the CMUT in Table I, in water ( $1 \mathrm{~atm})$, under $60 \mathrm{~V}$ bias voltage, and $0.1 \mathrm{~V}$ ac excitation.

velocities are the same as seen in Fig.2. The difference is at most $2.5 \%$ at the peak point.

We observed that $C_{R m_{-} a c}$ with a positive value has a strong hardening effect, and the resonance frequency is not predicted correctly when it is ignored.

\section{Mutual Radiation Impedance of Cells in Collapsed Mode}

In the collapsed mode, the mutual radiation impedance between two CMUT cells depends on the contact radii of both CMUTs. In order to find the mutual radiation impedance of two CMUTs with the same or different contact radii, first the velocity profile of the two collapsed mode cells are approximately calculated using uniform pressure formula of Timoshenko [4]. Then, a summation of four velocity profiles, for which the pressure calculations are analytically defined, are combined with weighting coefficients to be fitted to the collapsed plate profile. Once a profile is expressed in the form of a fourth order polynomial, the pressure field on the other surface can be directly calculated using the formulation of [5]. The pressure applied on the second CMUT is integrated over the plate surface in order to obtain the total force. Then the mutual impedance is calculated by dividing the total force by the rms velocity ${ }^{2}$ of the first CMUT.

In Fig. 3, the normalized mutual radiation impedance is plotted as a function of $k d$ for $k a=1$, where $k$ is the

$$
{ }^{2} v_{R}=1 /\left(\pi a^{2}\right) \sqrt{\int_{0}^{a} 2 \pi r v^{2}(r) d r}
$$


wavenumber, and $d$ is the distance between the centers of the CMUTs, and the mutual impedance is given by

$$
Z_{12}=\pi a^{2} \rho_{0} c_{0}\left\{R_{12}+j X_{12}\right\} .
$$

where $\rho_{0}$ is density and $c_{0}$ is the speed of sound in the immersion liquid. The normalized contact radius $\left(\bar{b}_{1}=b_{1} / a\right)$ of the first CMUT is fixed to 0.3 , and there are four different plots for $0,0.2,0.4,0.6$ normalized contact radii of the second CMUT. At a fixed operation frequency, the mutual radiation impedance depends on $d$, and it assumes both positive and negative values as $d$ changes. This is because the phase of the pressure created by a CMUT varies with the distance, and it may positively or negatively effect the operation of a neighboring CMUT, depending on the inter-cell distance. Hence, the enforcing and blocking behavior are observed continuously at multiples of half wavelength.

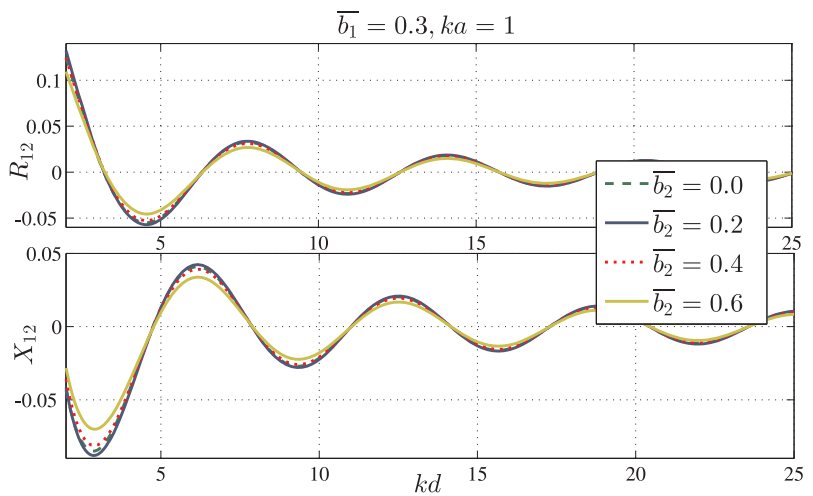

Fig. 3. The mutual radiation impedance as a function of $k d$, at different values of $\overline{b_{2}}$ for $\overline{b_{1}}=0.3, k a=1$.

Note that, impedance magnitude change is not significant although the contact radius is allowed to reach $0.6 a$. We also observe that the mutual radiation impedance is slowly decaying function, resulting in interactions of all cells even in large size arrays.

The mutual radiation impedance is plotted in Fig. 4 as a function of $k d$ for $d=5 a$. Here, we actually see how the mutual radiation impedance changes with frequency.

\section{Collapsed Mode Arrays}

Using both the large signal and the small signal models, we simulated the experimental results of a study carried out by Park et. al. [6] (Table II). In this work, a CMUT array of $64 \times 40$ cells is operated in collapsed mode at $10 \mathrm{MHz}$, at different bias levels ranging from $10 \mathrm{~V}$ to $180 \mathrm{~V}$. The same bias voltage is applied to all cells, while only a single element of $1 \times 40$ cells is driven with $\mathrm{AC}$ voltage, the remaining neighbors are inactive.

Interactions of every CMUT pair, including inactive cells, are considered in the model. Fig. 5 shows the mutual radiation impedance at different contact radii as a function of $k d$. At $180 \mathrm{~V}$ bias, $\bar{b}=0.45$. In the simulation range $\bar{b}=0$ to 0.45 , the mutual radiation impedance is almost the same for $k a=1.25$

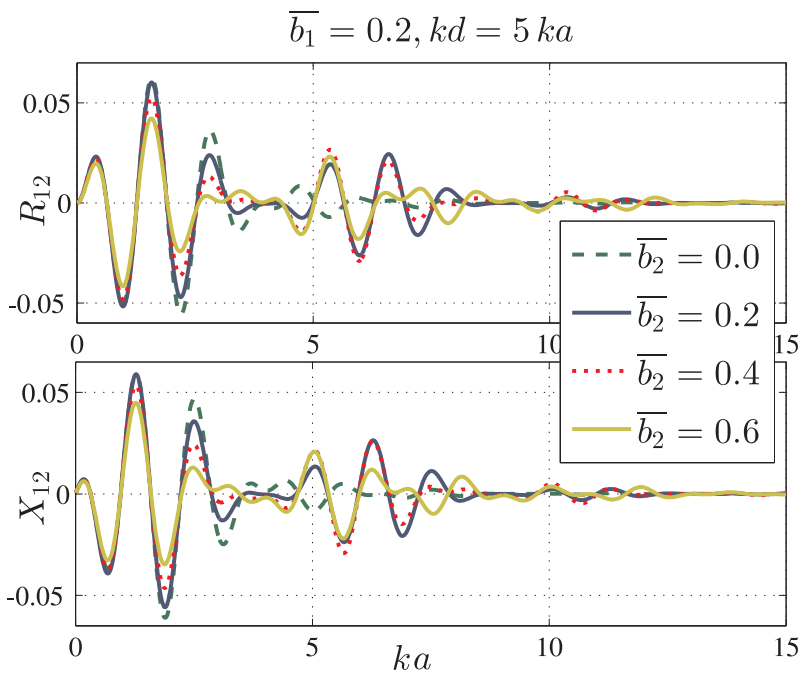

Fig. 4. The mutual radiation impedance as a function of $k d$ for $d=5 a$.

\begin{tabular}{|l|l|l|l|l|}
\hline$E$ & $v$ & $\epsilon_{r}$ & $\rho$ & P \\
\hline $148 \mathrm{GPa}$ & 0.1773 & 3.7 & $2.329 \mathrm{~g} / \mathrm{cm}^{3}$ & $1 \mathrm{~atm}$ \\
\hline \hline$a$ & $d$ & $t_{m}$ & $t_{i}$ & $t_{g}$ \\
\hline $30 \mu \mathrm{m}$ & $65 \mu \mathrm{m}$ & $1 \mu \mathrm{m}$ & $0.11 \mu \mathrm{m}$ & $0.5 \mu \mathrm{m}$ \\
\hline \multicolumn{5}{|c|}{ TABLE II }
\end{tabular}

PARAMETERS OF THE CMUT CELL USED IN SIMULATIONS

( $k a$ value at $10 \mathrm{MHz}$ ). One observation is that the most negative impedance is reached at $\bar{b}=0.2$ instead of $\bar{b}=0$.

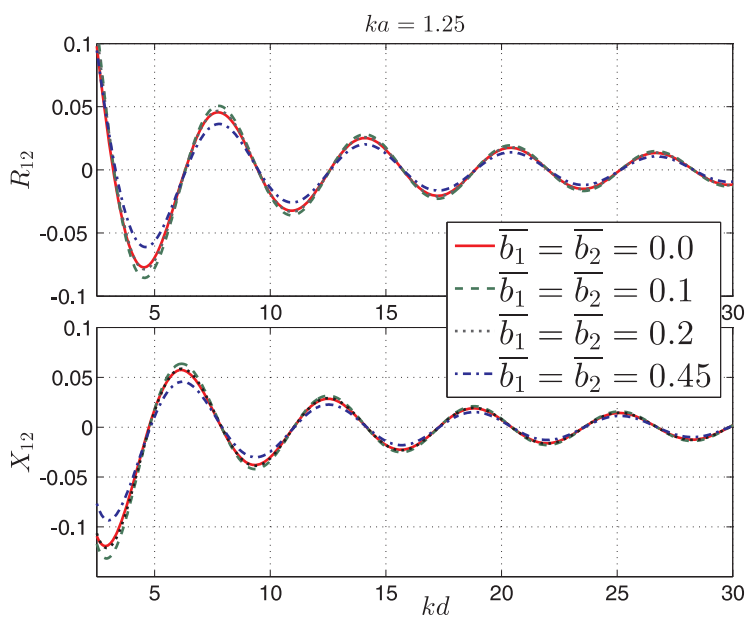

Fig. 5. The mutual radiation impedance plots for two CMUTs with the same contact radius for $k a=1.25$.

Fig. 6 depicts the measurement results of [6], and the lumped element analysis of the corresponding CMUT element with different number of passive neighbors included in the simulation. We observe that the passive neighbors are affective on the active cell behavior, and with increasing number of neighbors, the simulations get closer to the measured data. 


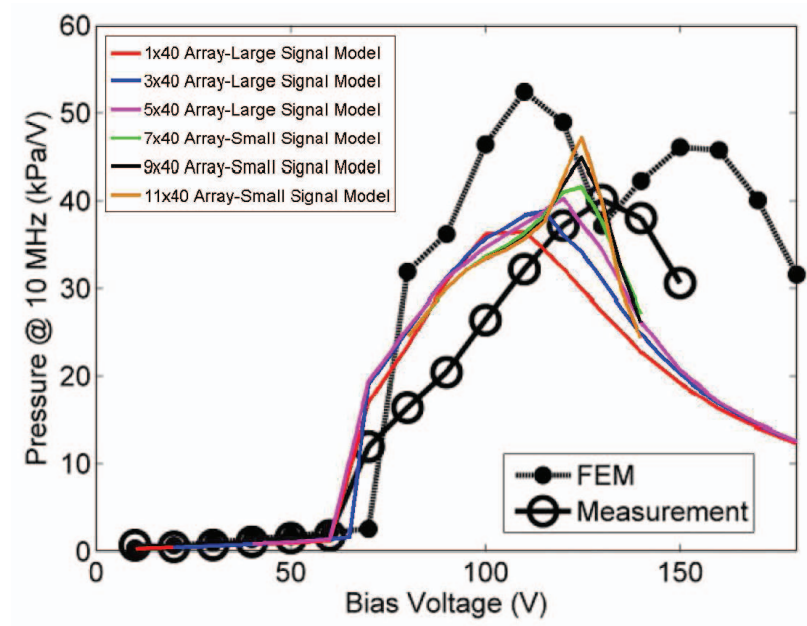

Fig. 6. The simulated and measured pressure obtained with a $1 \times 40$ element with neighboring inactive elements, at $10 \mathrm{MHz}$ at different bias voltages.

Fig. 7 depicts the simulated acoustic power output of a $21 \times 40$ element as a function of frequency. The curve is very similar to the measurement results given in [6]. We introduced a loss of about $1.2 \mathrm{~dB}$ in the mechanical side, which killed a resonance appearing around $6 \mathrm{MHz}$ due to interactions between the cells.

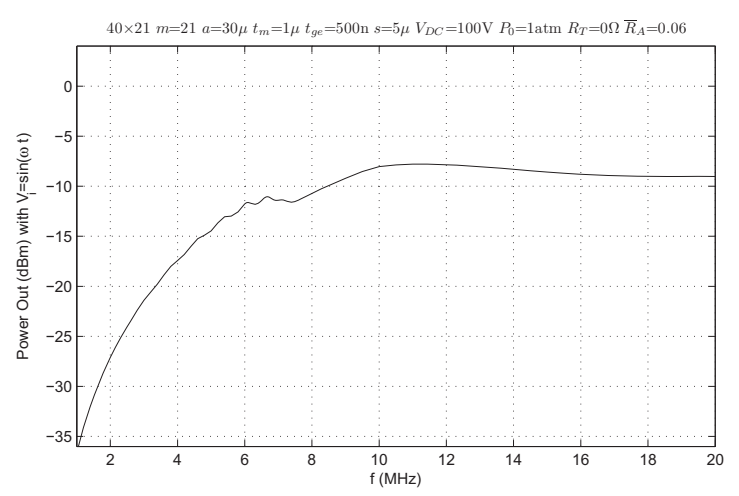

Fig. 7. The simulated power output at different frequencies obtained with a 21 element $21 \times 40$ array with only center element excited while all the other elements are shorted ac-wise, at $100 \mathrm{~V}$ bias voltage.

To be able to compare the performance of uncollapsed and collapsed CMUT cells, we simulate two CMUT elements each containing $1 \times 40$ CMUT cells with a radius of $a=30 \mu \mathrm{m}$. The first element operates in the conventional uncollapsed mode with a collapse voltage of $130 \mathrm{~V}$, while the second element is made from cells operating in collapse mode with a collapse voltage of $62 \mathrm{~V}$. Both elements are biased with $100 \mathrm{~V}$ and are driven by a $1 \mathrm{~V}$ peak $50 \Omega$ ac source. To keep the center frequency nearly the same, the first element has cells with a plate thickness of $4 \mu \mathrm{m}$, while the second element has $1 \mu \mathrm{m}$. Fig. 8 shows the results together with the simulation parameters. The element with collapsed cells produces about
$6 \mathrm{~dB}$ more acoustic power output. This predicted difference is consistent with FEM results reported earlier [6]. We note that our model only accounts for the fundamental vibration mode of the plate, it fails at high frequencies where higher order plate modes can be excited. Our model is derived for

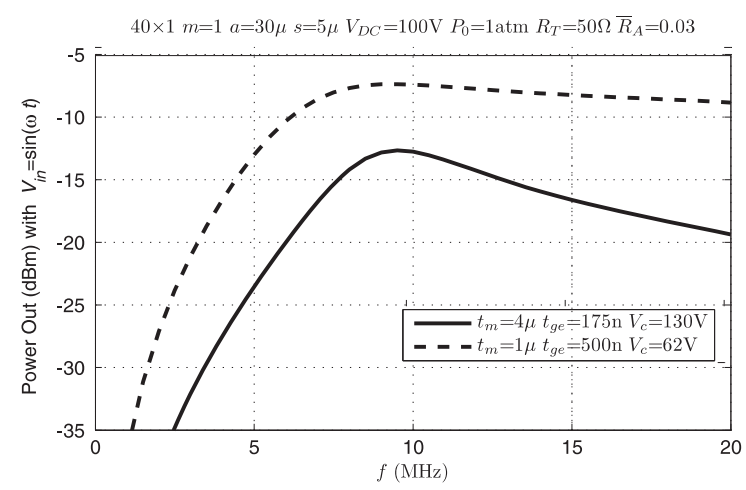

Fig. 8. The output power as a function of frequency.

fully electroded cells whereas the half electroded cells are used in [6]. This difference results in some mismatch between our predictions and the measurement data, particularly at high bias voltages where the contact radius is larger.

\section{CONClusion}

A parametric simulation tool for CMUT arrays including both the collapsed and uncollapsed mode operations is obtained. In order to decrease the computation cost, a small signal lumped element model is obtained by linearizing the large signal model at the operation point. The simulations performed with the small signal model are consistent with the large signal model while the computation cost is reduced 30-50 times. Simulations with the large and small signal models successfully predicted the measurements of a fabricated CMUT array.

\section{REFERENCES}

[1] H. K. Oguz, A. Atalar, and H. Koymen, "Equivalent circuit-based analysis of CMUT cell dynamics in arrays," IEEE Trans. Ultrason., Ferroelectr., Freq. Control, vol. 60, pp. 1016-1023, 2013.

[2] E. Aydogdu, A. Ozgurluk, A. Atalar, and H. Koymen, "Parametric nonlinear lumped element model for circular CMUTs in collapsed mode," IEEE Trans. Ultrason., Ferroelectr., Freq. Control, vol. 61, pp. 173-181, 2014.

[3] H. Koymen, A. Atalar, E. Aydogdu, C. Kocabas, H. K. Oguz, S. Olcum, A. Ozgurluk, and A. Unlugedik, "An improved lumped element nonlinear circuit model for a circular CMUT cell," IEEE Trans. Ultrason., Ferroelectr., Freq. Control, vol. 59, pp. 1791-1799, 2012.

[4] S. Timoshenko and S. W. Woinowsky-Krieger, Theory of Plates And Shells. NY: McGraw Hill, 1959.

[5] D. T. Porter, "Self and mutual radiation impedance and beam pat- terns for fexural disks in a rigid plane," J. Acoust. Soc. Am., vol. 36, pp. 1154 1161, 1964.

[6] K. K. Park, O. Oralkan, and B. T. Khuri-Yakub, "A comparison between conventional and collapse-mode capacitive micromachined ultrasonic transducers in 10-MHz 1-D arrays," IEEE Trans. Ultrason., Ferroelectr., Freq. Control, vol. 60, pp. 1245-1255, 2013. 